\title{
Complete convergence for weighted sums of arrays of rowwise
}

\section{negatively dependent random variables}

\author{
Bing Meng ${ }^{1,2, a}$, Dingcheng Wang ${ }^{3,1, b}$
}

${ }^{1}$ School of Mathematical Science, University of Electronic Science and Technology of China, Chengdu, Sichuan 611731, China

${ }^{2}$ College of Science, Guilin University of Technology, Guilin 541004, China

${ }^{3}$ Center of Financial Engineering, Nanjing Audit University, Nanjing 211815, China

a mengbing735@163.com, ${ }^{\mathrm{b}}$ wangdc@uestc.edu.cn

Abstract In this paper, we further study some sufficient conditions for complete convergence for weighted sums of arrays of rowwise negatively dependent random variables with non-identical distribution under some weaker moment conditions. Our result generalize and improve the corresponding result of Wang et al. [7].

Keywords: negatively dependent random variables, complete convergence, weighted sums

\section{Introduction}

Definition 1.1 A finite collection of random variables $X_{1}, X_{2}, \cdots, X_{n}$ is said to be negatively dependent (ND) if both

$$
P\left(X_{1}>x_{1}, X_{2}>x_{2}, \cdots, X_{n}>x_{n}\right) \leq \prod_{i=1}^{n} P\left(X_{i}>x_{i}\right)
$$

and

$$
P\left(X_{1} \leq x_{1}, X_{2} \leq x_{2}, \cdots, X_{n} \leq x_{n}\right) \leq \prod_{i=1}^{n} P\left(X_{i} \leq x_{i}\right)
$$

hold for all real numbers $x_{1}, X_{2}, \cdots, x_{n}$. An infinite sequence $\left\{X_{n}, n \geq 1\right\}$ is said to be ND if every finite subcollection is negatively dependent.

In the past decades, many authors have studied this concept and provided some interesting results and applications. For example, we refer to [2, 4, 5, 6]. Recently, Wang et al. [7] obtained the following complete convergence result for weighted sums of ND random variables with identical distribution.

Theorem 1.1 Let $\left\{X_{n i}, i \geq 1, n \geq 1\right\}$ be an array of row-wise ND random variables which is stochastically dominated by a random variable $X$, and $\left\{a_{n i}, i \geq 1, n \geq 1\right\}$ be an array of constants such that $\sum_{i=1}^{n}\left|a_{n i}\right|^{\alpha}=O\left(n^{\delta}\right)$ for some $\alpha$ with $0<\alpha<2$ and some $\delta$ with 
$0<\delta<1$. Assume further that $E X_{n i}=0$ when $1<\alpha<2$. If for some $h>0$ and $\gamma>0$ such that

$$
E\left\{\exp \left(h|X|^{\gamma}\right)\right\}<\infty
$$

then for any $\varepsilon>0$,

$$
\sum_{n=1}^{\infty} n^{p \alpha-2} P\left(\max _{1 \leq j \leq n}\left|\sum_{i=1}^{j} a_{n i} X_{n i}\right|>\varepsilon b_{n}\right)<\infty .
$$

where $p \geq 1 / \alpha$ and $b_{n}=n^{1 / \alpha}(\log n)^{1 / \gamma}$.

Inspired by the above theorem obtained by Wang [7], in this work, we will further study the compete convergence for weighted sums of arrays of rowwise ND random variables under some mild moment conditions, which are weaker than the above Theorem 1.1. Some complete convergence for the maximum weighted sums of arrays of rowwise ND random variables are obtained without the assumption of identical distribution. The result generalize and improve the corresponding result of Wang et al. [7].

\section{Main result and proof}

Throughout this paper, $C$ will represent a generic positive constant whose value may change from one appearance to the next, and $a_{n}=O\left(b_{n}\right)$ will mean $a_{n} \leq C b_{n}$. Let $I(A)$ be the indicator function of the set $A$.

Definition 2.1 A sequence of random variables $\left\{X_{n}, n \geq 1\right\}$ is said to be stochastically dominated by a random variable $X$ if there exists a positive constant $C$ such that $P\left(\left|X_{n}\right| \geq x\right) \leq C P(|X| \geq x)$ for all $x \geq 0$ and $n \geq 1$.

Lemma 2.1 [3] Let $X_{1}, X_{2}, \cdots, X_{n}$ be ND random variables and $f_{1}, f_{2}, \cdots, f_{n}$ be a sequence of Borel functions which all are monotone increasing (or all are monotone decreasing), then $f_{1}\left(X_{1}\right), f_{2}\left(X_{2}\right), \cdots, f_{n}\left(X_{n}\right)$ are ND random variables.

Lemma 2.2 [8] Let $p \geq 2$ and $\left\{X_{n}, n \geq 1\right\}$ be a sequence of ND random variables with $E X_{n}=0$ and $E\left|X_{n}\right|^{p}<\infty$ for every $n \geq 1$. Then, there exists a positive constant $C$ depending only on $p$ such that for every $n \geq 1$,

$$
E\left(\max _{1 \leq j \leq n}\left|\sum_{i=1}^{j} X_{i}\right|^{p}\right) \leq C \log ^{p} 2 n\left\{\sum_{i=1}^{n} E\left|X_{i}\right|^{p}+\left(\sum_{i=1}^{n} E X_{i}^{2}\right)^{p / 2}\right\}
$$


Lemma 2.3 [1] Let $\left\{X_{n}, n \geq 1\right\}$ be a sequence of random variables which is stochastically dominated by a random variable $X$. For any $u>0, t>0$ and $n \geq 1$, the following two statements hold:

$$
\begin{gathered}
E\left|X_{n}\right|^{u} I\left(\left|X_{n}\right| \leq t\right) \leq C\left[E|X|^{u} I(|X| \leq t)+t^{u} P(|X|>t)\right] \\
E\left|X_{n}\right|^{u} I\left(\left|X_{n}\right|>t\right) \leq C E|X|^{u} I(|X|>t) .
\end{gathered}
$$

Theorem 2.1 Let $\left\{X_{n i}, i \geq 1, n \geq 1\right\}$ be an array of row-wise ND random variables which is stochastically dominated by a random variable $X$ and $\left\{a_{n i}, i \geq 1, n \geq 1\right\}$ be an array of real numbers. Assume that there exist some $\delta$ with $0<\delta<1$ and some $\alpha$ with $0<\alpha \leq 2$ such that $\sum_{i=1}^{n}\left|a_{n i}\right|^{\alpha}=O\left(n^{\delta}\right)$, and assume further that $E X_{n i}=0$ when $1<\alpha \leq 2$. If there exists

$$
\beta>\max \left\{\alpha+2, \alpha^{2} s, \frac{\alpha(s \alpha-1)}{1-\delta}, \alpha(s \alpha-1)+2 \delta\right\}
$$

and $s \alpha \geq 1$ such that $E|X|^{\beta}<\infty$, then for any $\varepsilon>0$,

$$
\sum_{n=1}^{\infty} n^{s \alpha-2} P\left(\max _{1 \leq j \leq n}\left|\sum_{i=1}^{j} a_{n i} X_{n i}\right|>\varepsilon b_{n}\right)<\infty,
$$

where $b_{n}=n^{1 / \alpha}(\log n)^{1 / \gamma}$ for some $\gamma>0$.

Proof For $\forall i \geq 1$, define

$$
\begin{gathered}
X_{i}^{(n)}=-b_{n} I\left(X_{n i}<-b_{n}\right)+X_{n i} I\left(\left|X_{n i}\right| \leq b_{n}\right)+b_{n} I\left(X_{n i}>b_{n}\right), \\
T_{j}^{(n)}=\sum_{i=1}^{j} a_{n i}\left(X_{i}^{(n)}-E X_{i}^{(n)}\right), \quad j=1,2, \cdots, n .
\end{gathered}
$$

It is easy to check that for $\forall \varepsilon>0$,

$$
\left\{\max _{1 \leq j \leq n}\left|\sum_{i=1}^{j} a_{n i} X_{n i}\right|>\varepsilon b_{n}\right\} \subset\left\{\max _{1 \leq i \leq n}\left|X_{n i}\right|>b_{n}\right\} \cup\left\{\max _{1 \leq j \leq n}\left|\sum_{i=1}^{j} a_{n i} X_{i}^{(n)}\right|>\varepsilon b_{n}\right\},
$$

which implies

$$
\begin{aligned}
& P\left(\max _{1 \leq j \leq n}\left|\sum_{i=1}^{j} a_{n i} X_{n i}\right|>\varepsilon b_{n}\right) \\
& \leq \sum_{i=1}^{n} P\left(\left|X_{n i}\right|>b_{n}\right)+P\left(\max _{1 \leq j \leq n}\left|T_{j}^{(n)}\right|>\varepsilon b_{n}-\max _{1 \leq j \leq n}\left|\sum_{i=1}^{j} a_{n i} E X_{i}^{(n)}\right|\right) .
\end{aligned}
$$


Firstly, we will prove that

$$
b_{n}^{-1} \max _{1 \leq j \leq n}\left|\sum_{i=1}^{j} a_{n i} E X_{i}^{(n)}\right| \rightarrow 0 \text { as } n \rightarrow \infty .
$$

For $1<\alpha \leq 2$, it follows from $E X_{n i}=0$, Lemma 2.3, the hölder inequality and the Markov inequality that

$$
\begin{aligned}
b_{n}^{-1} \max _{1 \leq j \leq n}\left|\sum_{i=1}^{j} a_{n i} E X_{i}^{(n)}\right| & \leq n b_{n}^{-\beta} E\left|X_{i}\right|^{\beta}+b_{n}^{-1} \sum_{i=1}^{n} E\left|a_{n i} X_{i}\right| I\left(\left|X_{i}\right|>b_{n}\right) \\
& \leq C n b_{n}^{-\beta}+C b_{n}^{-1} n \sum_{k=n}^{\infty} E|X| I\left(b_{k}<|X| \leq b_{k+1}\right) \\
& \leq C n b_{n}^{-\beta}+C b_{n}^{-1} n \sum_{k=n}^{\infty} b_{k+1} P\left(|X|>b_{k}\right) \\
& \leq C n b_{n}^{-\beta}+C b_{n}^{-1} n \sum_{k=n}^{\infty} b_{k+1} \frac{E|X|^{\beta}}{b_{k}^{\beta}} \\
& \leq \frac{C n}{n^{\beta / \alpha} \log ^{\beta / \gamma} n}+C b_{n}^{-1} \sum_{k=n}^{\infty} k^{1 / \alpha+1-\beta / \alpha} \\
& \leq \frac{C n^{1-\beta / \alpha}}{\log ^{\beta / \gamma} n}+\frac{C n^{2-\beta / \alpha}}{\log ^{1 / \gamma} n} \rightarrow 0, \text { as } n \rightarrow \infty
\end{aligned}
$$

For $0<\alpha \leq 1$, it follows from Lemma 2.3, the Jensen inequality and the Markov inequality again that

$$
\begin{aligned}
b_{n}^{-1} \max _{1 \leq j \leq n}\left|\sum_{i=1}^{j} E a_{n i} X_{i}^{(n)}\right| & \leq C b_{n}^{-1} \sum_{i=1}^{n}\left|a_{n i}\right| E\left|X_{n i}\right| I\left(\left|X_{n i}\right| \leq b_{n}\right)+C \sum_{i=1}^{n}\left|a_{n i}\right| P\left(\left|X_{n i}\right|>b_{n}\right) \\
& \leq C b_{n}^{-1} n^{\delta / \alpha} E|X| I\left(|X| \leq b_{n}\right)+C n^{\delta / \alpha} P\left(|X|>b_{n}\right) \\
& \leq C b_{n}^{-1} n^{\delta / \alpha} \sum_{k=2}^{n} E|X| I\left(b_{k-1}<|X| \leq b_{k}\right)+C n^{\delta / \alpha} P\left(|X|>b_{n}\right) \\
& \leq C b_{n}^{-1} n^{\delta / \alpha} \sum_{k=2}^{n} b_{k} E|X|^{\beta} b_{k-1}^{-\beta}+C n^{\delta / \alpha} b_{n}^{-\beta} E|X|^{\beta} \\
& \leq \frac{C n^{\delta / \alpha} n^{1 / \alpha-\beta / \alpha+1}}{n^{1 / \alpha} \log ^{1 / \gamma} n}+\frac{C n^{\delta / \alpha}}{n^{\beta / \alpha} \log ^{\beta / \gamma} n} \rightarrow 0 \text {, as } n \rightarrow \infty
\end{aligned}
$$

Hence, to prove (2.5), it suffices to show that 


$$
\begin{gathered}
I \triangleq \sum_{n=1}^{\infty} n^{\alpha s-2} \sum_{i=1}^{n} P\left(\left|X_{n i}\right|>b_{n}\right)<\infty, \\
J \triangleq \sum_{n=1}^{\infty} n^{\alpha s-2} P\left(\max _{1 \leq j \leq n}\left|T_{j}^{(n)}\right|>\frac{\varepsilon b_{n}}{2}\right)<\infty .
\end{gathered}
$$

In fact, by the Markov inequality, we get that

$$
I \leq C \sum_{n=1}^{\infty} n^{\alpha s-2} \sum_{i=1}^{n} P\left(|X|>b_{n}\right) \leq C \sum_{n=1}^{\infty} n^{\alpha s-1} n^{-\beta / \alpha} \log ^{-\beta / \gamma} n<\infty .
$$

Hence, for $q>2$, it follows from Lemma 2.2 and the Jensen inequality that

$$
\begin{aligned}
& J \leq C \sum_{n=1}^{\infty} n^{\alpha s-2} b_{n}^{-q} E\left(\max _{1 \leq j \leq n}\left|T_{j}^{(n)}\right|^{q}\right) \\
& \leq C \sum_{n=2}^{\infty} n^{\alpha s-2} b_{n}^{-q}(\log n)^{q} \sum_{i=1}^{n}\left|a_{n i}\right|^{q} E\left|X_{i}^{(n)}\right|^{q}+C \sum_{n=2}^{\infty} n^{\alpha s-2} b_{n}^{-q}(\log n)^{q}\left(\sum_{i=1}^{n}\left|a_{n i}\right|^{2} E\left|X_{i}^{(n)}\right|^{2}\right)^{q / 2} \\
& \triangleq J_{1}+J_{2}
\end{aligned}
$$

Take a suitable constant $q$ such that

$$
\max \left\{2, \frac{\alpha(s \alpha-1)}{1-\delta}\right\}<q<\min \left\{\beta-\alpha, \frac{\beta+\alpha-\alpha^{2} s}{\delta}\right\}
$$

which implies

$$
\beta>\alpha+q, \frac{\beta}{\alpha}-\frac{q}{\alpha}>1, \beta>q \delta-\alpha+\alpha^{2} s, \alpha s-2+\frac{q \delta}{\alpha}-\frac{q}{\alpha}<-1, q>\alpha .
$$

It follows from the Jensen inequality and Lemma 2.3 that

$$
\begin{aligned}
J_{1} \leq & C \sum_{n=2}^{\infty} n^{\alpha s-2} b_{n}^{-q}(\log n)^{q} \sum_{i=1}^{n}\left|a_{n i}\right|^{q}\left(E\left|X_{n i}\right|^{q} I\left(\left|X_{n i}\right| \leq b_{n}\right)+b_{n}^{q} P\left(\left|X_{n i}\right|>b_{n}\right)\right) \\
\leq & C \sum_{n=2}^{\infty} n^{\alpha s-2+q \delta / \alpha} b_{n}^{-q}(\log n)^{q} \sum_{k=2}^{n} E|X|^{q} I\left(b_{k-1}<|X| \leq b_{k}\right) \\
& +C \sum_{n=2}^{\infty} n^{\alpha s-2+q \delta / \alpha}(\log n)^{q} b_{n}^{-\beta} E|X|^{\beta} \\
\leq & C \sum_{k=2}^{\infty} b_{k}^{q} P\left(|X|>b_{k-1}\right) \sum_{n=k}^{\infty} n^{\alpha s-2+q \delta / \alpha-q / \alpha}(\log n)^{q-q / \gamma}+C \sum_{n=2}^{\infty} \frac{n^{\alpha s-2+q \delta / \alpha}(\log n)^{q}}{n^{\beta / \alpha}(\log n)^{\beta / \gamma}} \\
\leq & C \sum_{k=3}^{\infty} \frac{k^{q / \alpha}(\log k)^{q / \gamma}}{(k-1)^{\beta / \alpha}(\log (k-1))^{\beta / \gamma}}+C \sum_{n=2}^{\infty} \frac{n^{\alpha s-2+q \delta / \alpha}(\log n)^{q}}{n^{\beta / \alpha}(\log n)^{\beta / \gamma}}<\infty .
\end{aligned}
$$


It follows from the $C_{r}$ inequality, Lemma 2.3 and the Jensen inequality that

$$
\begin{aligned}
J_{2} & \leq C \sum_{n=2}^{\infty} n^{\alpha s-2} b_{n}^{-q}(\log n)^{q}\left(\sum_{i=1}^{n} a_{n i}^{2}\left(E X_{n i}^{2} I\left(\left|X_{n i}\right| \leq b_{n}\right)+b_{n}^{2} P\left(\left|X_{n i}\right|>b_{n}\right)\right)\right)^{q / 2} \\
& \leq C \sum_{n=2}^{\infty} n^{\alpha s-2} b_{n}^{-q}(\log n)^{q}\left(\sum_{i=1}^{n} a_{n i}^{2}\left(E X^{2} I\left(|X| \leq b_{n}\right)+b_{n}^{2} P\left(|X|>b_{n}\right)\right)\right)^{q / 2} \\
& \leq C \sum_{n=2}^{\infty} n^{\alpha s-2} b_{n}^{-q} n^{q \delta / \alpha}(\log n)^{q} E|X|^{q} I\left(|X| \leq b_{n}\right)+C \sum_{n=2}^{\infty} n^{\alpha s-2} n^{q \delta / \alpha}(\log n)^{q} P\left(|X|>b_{n}\right) \\
& <\infty \text { (see the proof of (2.15)). }
\end{aligned}
$$

This completes the proof of the theorem 2.1.

\section{Acknowledgments}

This research is supported by the National Natural Science Foundation of China (71271042; 11661029; 11661030; 71501025), Applied Basic Project of Sichuan Province (2016JY0257). Corresponding author: Dingcheng Wang. E-mail: wangdc@uestc.edu.cn

\section{References}

[1] Adler, A., Rosalsky, A.. Some general strong laws for weighted sums of stochastically dominated random variables. Stochastic Anal. Appl. 1987, 5: 1-16.

[2] Asadian, N., Fakoor, V., Bozorgnia, A.. Rosental's type inequalities for negatively orthant dependent random variables. J. Iran. Stat. Soc. 2006, 5(1-2): 66-75.

[3] Bozorgnia, A., Patterson, R.F., Taylor, R.L.. Limit theorems for dependent random variables. World Congress Nonlinear Analysts. 1996, 92: 1639-1650.

[4] Ko, MH., Kim, TS.. Almost sure convergence for weighted sums of negatively orthant dependent random variables. J. Korean Math. Soc. 2005, 42(5): 949-957.

[5] Qiu, D.H., Wu, Q.y., Chen, P.Y.. Complete convergence for negatively orthant dependent random variables. J. Ineq. Appl. 2014, 145.

[6] Qiu D.H., Chang K.C., Antonini, R.G., Volodin, A. On the Strong Rates of Convergence for Arrays of Rowwise Negatively Dependent Random Variables. Stoch. Anal. Appl. 2011, 29(3): 375-385.

[7] Wang, X.J., Hu, S.H., Yang, W.Z.. Complete convergence for arrays of rowwise negatively orthant dependent random variables. RACSAM. 2012, 106:235 - 245.

[8] Wu Q.Y.. Complete convergence for weighted sums of sequences of negatively dependent random variables. J. Probab. Stat. 2011, Article ID 202015. 